\title{
Émile Goichot (éd.), Luigi Sturzo e gli intellettuali catolici francesi Carteggi (1925-1945)
}

Rome, Institut Luigi Sturzo, 2004, XII+458 p. (coll. « Opera omnia di Luigi Sturzo », vol. IV-7)

Émile Poulat

\section{OpenEdition}

Journals

Édition électronique

URL : http://journals.openedition.org/assr/2564

DOI : $10.4000 /$ assr.2564

ISSN : $1777-5825$

Éditeur

Éditions de l'EHESS

Édition imprimée

Date de publication : 1 octobre 2004

Pagination : 53-158

ISBN : 2-222-96754-6

ISSN : 0335-5985

Référence électronique

Émile Poulat, «Émile Goichot (éd.), Luigi Sturzo e gli intellettuali catolici francesi Carteggi (1925-1945) 》, Archives de sciences sociales des religions [En ligne], 128 | octobre - décembre 2004, document 128.62 mis en ligne le 18 novembre 2005, consulté le 24 septembre 2020. URL : http:// journals.openedition.org/assr/2564; DOI : https://doi.org/10.4000/assr.2564 
de la fin du XVIII ${ }^{e}$ siècle, écrivait naguère : «Chaque région a sa folie. La Bretagne les a toutes. »?

Nul ne contestera que, deux siècles plus tard, les choses ont quelque peu changé. La Bretagne d'aujourd'hui est, en bien des domaines, à l'avant-garde du monde rural français et les producteurs agricoles du pays Léonard ou des Côtes d'Armor se soucient plus des directives émanant de Bruxelles que des loups-garous et autres messagers du malheur.

Toutefois, dans un ouvrage magnifiquement illustré - à ce seul titre, il mériterait de retenir l'attention - nos auteurs évoquent avec bonheur tout un univers de croyances et de légendes où voisinent en bonne intelligence mégalithes et fontaines, chapelles et saints guérisseurs, sorciers et rebouteux, revenants et animaux porte malheur, sans oublier ces mystérieux « intersignes » où s'annonce la mort.

Sont-ce là les survivances d'un monde ancien qui cherchait tant bien que mal à se défendre de la maladie ou de la stérilité ? Ce n'est pas si sûr car il arrive encore aujourd'hui qu'une femme en mal d'enfant aille solliciter l'aide du menhir qui se dresse à la lisière d'un champ ou l'eau d'une fontaine tenue pour miraculeuse. Au total, disent nos AA. «mille et une croyances dont certaines sont encore bien vivaces aujourd'hui. » (p. 58). Cela n'est-il vrai qu'en Bretagne? À l'évidence, non! Y-a-t-il lieu de s'en étonner? Pas davantage. Dans l'Airbus 380 que nous concocte aujourd'hui la fine pointe de la technologie moderne, il n'y aura pas de rangée 13 . Trop de passagers refuseraient d'y être assis ! Le 13 porte malheur, sauf les vendredis...

Un regret toutefois. II manque à ce livre, à bien des égards remarquable, une carte de la Bretagne et de ses haut-lieux de mémoire! Elle aurait été fort utile.

\section{René Luneau.} MILLER (Donald E.), éds.

GenX religion. New York-Londres, Routledge, 2000, 257 p. (index).

La Génération $X$ désigne quatre-vingt millions d'Américains, nés entre 1961 et 1981. Enfants des baby-boomers, ils ont vécu les progrès technologiques, les changements politiques et économiques ainsi que la déstructuration de la cellule familiale qui ont affecté la société américaine de la fin du vingtième siècle. En ce qui concerne la religion, cette génération que les médias décrivent comme une jeunesse apathique, cynique et insatisfaite, aurait pour caractéristique un faible investissement dans les Églises traditionnelles.

Toutefois, ce recueil veut montrer que la réticence de la Génération X à s'engager dans les structures religieuses et dénominations traditionnelles ne signifie pas pour autant qu'elle se désintéresse des questions de sens et de valeurs. $\mathrm{Au}$ contraire, la Génération X manifesterait une recherche spirituelle au sein de nouvelles institutions et dans un environnement religieux réinventé. Les contributions de cet ouvrage illustrent la diversification des organisations religieuses ciblant la jeune génération : salons de tatouage évangéliques, milieu gothique, service baptiste dans un night-club de Los Angeles, Églises charismatiques et pentecôtistes utilisant la culture des gangs et du hip-hop pour attirer les jeunes latino...

En conclusion, les auteurs résument les caractéristiques générales de la religion de la Génération X. Toutefois, les travaux ethnographiques présentés ici concernent exclusivement la Californie qui, faut-il noter, a été un lieu particulier d'innovations religieuses depuis les années soixante au moins (implantation du bouddhisme zen et de nouveaux mouvements religieux, développement du Potentiel Humain...). La génération $X$, se détournant des structures rigides auxquelles elle aurait à s'adapter, est déterminée à trouver un espace culturel dans lequel créer de nouvelles expressions religieuses. La religiosité de la Génération $X$ privilégierait tout particulièrement une expression sensible et expérientielle des croyances, utilisant textes, images, musiques, danses et expressions corporelles. Si elle partage avec les baby-boomers la valorisation d'une quête spirituelle, elle se distingue de ses aînés en ce qu'elle enracinerait cette quête dans une appartenance communautaire plutôt que dans une démarche purement personnelle. L'intégration «raciale» et les lois de discrimination positive mises en place par les baby-boomers font de la Génération X la génération du multiculturalisme. Aussi les AA. notent que la diversité ethnique et l'inclusivité se manifestent comme des objectifs explicites des formes de religion que cette génération privilégie.

$$
\text { Véronique Altglas. }
$$
GOICHOT (Émile), éd.

Luigi Sturzo e gli intellettuali catolici francesi Carteggi (1925-1945). Rome, Institut Luigi Sturzo, 2004, XII+458 p. (coll. «Opera omnia di Luigi Sturzo », vol. IV-7).

Que le lecteur français ne se laisse pas rebuter par le titre de cet ouvrage, en italien et en 
demi-teinte. En italien à la fois parce que le personnage clé de cette correspondance est un Sicilien et parce que son éditeur est à Rome. Demi-teinte parce que ses correspondants français ne représentent que l'aile gauche des intellectuels catholiques de France, et même pas tout entière : en clair, l'antifascisme démocratechrétien, engagé très tôt et sans équivoque dans la résistance aux totalitarismes.

Don Luigi Sturzo (1881-1951), ordonné prêtre en 1894, maire de son village natal en Sicile, Caltagirone, en 1905, a été un démocratechrétien de la première heure - génération Léon XIII -, avec Romolo Murri. À la différence de celui-ci, il sortira indemne de la « crise moderniste » dans laquelle se trouvèrent pris les novateurs. En janvier 1919, il lança le célèbre appel « Ai uomini liberi e forti... », aux hommes libres et courageux... - qui fonda le Parti populaire italien (PPI) dont il fut le secrétaire général. II dut s'exiler à Londres, puis à New York après 1'assassinat par une bande fasciste de Giacomo Matteotti, secrétaire général du Parti socialiste, le $1^{\text {er }}$ juin 1924 . II ne put rentrer dans son pays qu'en 1946. Un exil de plus de vingt ans, où il déploya une inlassable activité internationale.

Ce gros livre, présenté avec soin et même élégance, rassemble près de 700 lettres ou billets à ses amis français et belges, en recourant très vite à leur langue. La publication a été assurée par É.G., professeur émérite de littérature moderne et contemporaine à la Faculté des lettres de l'Université Marc-Bloch (Strasbourg), trop prématurément disparu voici un an. L'annotation est sobre, par volonté de ne pas épaissir le volume d'une érudition indigeste. Les introductions sont bilingues et disent l'essentiel. On y reconnaît un homme qui aimait l'Italie et qui était devenu un familier de sa culture.

La correspondance est classée chronologiquement, mais par familles d'amis: Marc Sangnier et le groupe de la Jeune République, Marcel Prélot et la famille de Maurice Blondel autour de la revue Politique, Élie Beaussart et son équipe belge de La Terre wallonne, Francisque Gay et le quotidien L'aube (de loin le chapitre le plus important), les dominicains des Éditions du Cerf et de La Vie intellectuelle, enfin, la guerre venue, les Maritains établis à New York et leur entourage.

On ne s'étonnera pas de ne pas y trouver les «chrétiens révolutionnaires » de Terre nouvelle. En revanche, on s'interrogera sur l'absence de relations avec Emmanuel Mounier et les personnalistes d'Esprit. II est vrai que Mounier ne cachait pas son dédain pour la démocratie chrétienne, ce «veau à deux têtes ». On notera enfin qu'il ne s'agit pas de relations

personnelles d'amitié, mais avec des groupes constitués. Entre eux, les rapports n'étaient pas toujours faciles : Sturzo apparaît comme un liant entre ces divers courants. Tous ceux-ci ont une connotation et un engagement politique : Sturzo semble n'avoir eu aucun contact avec les mouvements catholiques de jeunesse ouvrière ou étudiante. On observera simplement que sur le même tronc, le «progressisme chrétien» n'est pas sorti de la démocratie chrétienne, mais de ces mouvements d'action catholique. Pour les premiers, le communisme, c'était les terribles années qui ont suivi la Révolution d'Octobre ; pour les jeunes chrétiens combattants, c'était Stalingrad, la résistance intérieure et sa fraternité.

Une correspondance, on aime ou on n'aime pas. Ça ne court pas comme un récit, ce n'est pas une fresque. C'est du décousu, du pointillisme au jour le jour, de l'impressionnisme par petites touches, et du plancton pour les historiens. C'est l'histoire qui se fait sur le ton de la confidence, qui donne du temps à la réflexion. On verra ici comment se forme une sensibilité politique en pensant d'une part à ce tripartisme de la Libération, éphémère conjonction de trois antifascismes, d'autre part à cette allergie mutuelle, jamais surmontée malgré quelques passerelles, entre démocratie chrétienne et gaullisme.

Émile Poulat.

128.63

\section{GOULLET (Monique), LOBRICHON (Guy), PALAZZO (Éric).}

\section{Le Pontifical de la curie romaine au XIII}

siècle. (texte latin; traduction et introduction par Monique Goullet, Guy Lobrichon, Éric Palazzo), Paris, Le Cerf, 2004, 407 p. (index) (coll. «Sources liturgiques » $\mathrm{n}^{0} 4$ ).

Dans un projet ambitieux et significatif du renouveau que connaissent les études d'histoire de la liturgie, l'ouvrage offre le texte du Pontifical utilisé par la curie romaine, au $\mathrm{XIII}^{\mathrm{e}}$ siècle, dans une édition bilingue précédée d'une introduction d'une vingtaine de pages.

La version latine du texte avait été établie en 1939 par Michel Andrieu, avec celle du Pontifical du XII ${ }^{e}$ siècle et de celui de Guillaume Durand de Mende (pourquoi ne pas lui avoir rendu justice dès la page de garde ?). Les auteurs ont simplement réintroduits dans le Pontifical du XIII ${ }^{\mathrm{e}}$ siècle ici édité certaines pièces liturgiques du Pontifical du XII ${ }^{\mathrm{e}}$ qui n'avaient pas été reprises. La traduction proposée permet de rendre plus largement accessible un type de sources resté l'apanage d'un milieu restreint de 